DPUR/TH/15

March, 2009

\title{
Massive Gravity with Mass Term in Three Dimensions
}

\author{
Masashi Nakasone and Ichiro Oda ${ }^{1}$ \\ Department of Physics, Faculty of Science, University of the Ryukyus, \\ Nishihara, Okinawa 903-0213, JAPAN
}

\begin{abstract}
We analyze the effect of the Pauli-Fierz mass term on a recently established, new massive gravity theory in three space-time dimensions. We show that the Pauli-Fierz mass term makes the new massive gravity theory non-unitary. Moreover, although we add the gravitational Chern-Simons term to this model, the situation remains unchanged and the theory stays non-unitary in spite that structure of the graviton propagator is greatly changed. Thus, the Pauli-Fierz mass term is not allowed to coexist with massgenerating higher-derivative terms in the new massive gravity.
\end{abstract}

\footnotetext{
${ }^{1}$ E-mail address: ioda@phys.u-ryukyu.ac.jp
} 


\section{Introduction}

It is known that gravitational interaction is naturally coupled to the stress-energy tensor just like the electro-magnetic interaction is done to the electro-magnetic current. Since the stress-energy tensor is generated by mass (as well as momentum) of particles, understanding the quantum-mechanical meaning of mass is a crucial step in developing a quantum theory of gravity. Although the graviton mediating the gravitational interaction is a massless particle in Einstein's general relativity, there was an old attempt to getting the massive graviton by Pauli and Fierz [1]. It seems that this attempt of giving mass to the graviton has some applications to recent developments of quantum gravity, string theory, brane world, and cosmology and so on $[2,3,4,5,6,7,8,9,10]$. In hindsight, this is not surprising since general relativity is almost the unique theory of massless spin 2 gravitational field whose universality class is determined by local general coordinate transformations, any infrared modification of general relativity cannot aviod introduction of some kind of mass for the graviton.

In an arbitrary space-time dimension, if we do not introduce the other matter fields, there is the unique mass-generating mechanism for the graviton, which is adding the Pauli-Fierz mass term to the Einstein-Hilbert action. This mechanism makes it possible to generate mass to the graviton in a Lorentz-covariant manner without the emergence of a non-unitary ghost. However, there is at least one serious drawback in the Pauli-Fierz massive gravity in that the massive gravity only makes sense as a free and linearized theory, so it is unclear how to obtain a diffeomorphism-invariant mass and interaction terms in this framework.

Recently, in three space-time dimensions there has been an interesting progress for obtaining a sensible interacting massive gravity theory [11]. This model has been shown to be equivalent to the Pauli-Fierz massive gravity at the linearized approximation level. A key idea in this model is that one adds higher-derivative curvature terms to the Einstein-Hilbert action with the wrong sign in such a way that the trace part of the stress-energy tensor associated with those higher-derivative terms is proportional to the original higher-derivative Lagrangian. More recently, this new massive gravity model in three dimensions has been studied from various viewpoints such as the unitarity and the impossibility of generalization to higher dimensions [12], the AdS black hole solutions [13] and the properties of linearized gravitational excitations in asymptotically AdS space-time [14].

As a peculiarity of three dimensions, there is an alternative mass-generating mechanism for the graviton: adding a topological term named the gravitational Chern-Simons term [15]. Thus, in three dimensions, in total there are three distinct mass-generating mechanisms for the graviton, those are, adding the Pauli-Fierz, the higher-derivative, and the gravitational Chern-Simons terms. It is then natural to ask what becomes of mass of the graviton if these three terms coexist in one theory ${ }^{2}$. Surprisingly, these seemingly innocuous models are not physically acceptable owing to the emergence of ghosts and/or tachyons.

The aim of this article is to show that the Pauli-Fierz mass term is not allowed to exist in

\footnotetext{
${ }^{2}$ In the case of gauge fields, the relation between the conventional Higgs mechanism and the topological mass-generating mechanism was investigated in $[16,17]$.
} 
the new massive gravity in three dimensions. In this connection, it has been already verified that there is a consistent, unitary massive gravity with three massive excitations in some parameter region between mass and the coefficient of gravitational Chern-Simons terms when the Pauli-Fierz term is added to the topologically massive gravity in three dimensions $[18,19]$.

In the next section, we clarify why there is an interacting unitary massive gravity theory only in three dimensions via the analysis of structure of the graviton propagator in a general space-time dimension. In the third section, we consider the case that the Pauli-Fierz mass term is added to the new massive gravity theory and examine if the original massive graviton stays unitary or not. Here we realize that the existence of the Pauli-Fierz mass term has a tendency to make the unitary modes change to ghosts and tachyons. In the final section, we study the most general massive gravity model where the gravitational Chern-Simons term is added to the model treated in the section 3. Again we will see that there appear ghosts in the mass spectrum, so that the Pauli-Fierz term cannot afford to exist in the new massive gravity theory in three dimensions.

\section{Propagator in a general higher-derivative gravity}

In this section, we wish to pursue a possibility of constructing a renormalizable, interactive, unitary model with higher-derivative terms for the massive graviton by studying structure of the graviton propagator. Even if it is well known that it is impossible to construct such an ideal model in four space-time dimensions [20], it is useful to clarify the meaning of the new massive gravity model by Bergshoeff et al. [11] in three dimensions.

The action with which we start is a higher-derivative gravity model [20, 12] without cosmological constant up to fourth-order in derivative in a general $D$ space-time dimensions 3 :

$$
S=\int d^{D} x \sqrt{-g}\left[\frac{1}{\kappa^{2}} R+\alpha R^{2}+\beta R_{\mu \nu} R^{\mu \nu}+\gamma\left(R_{\mu \nu \rho \sigma} R^{\mu \nu \rho \sigma}-4 R_{\mu \nu} R^{\mu \nu}+R^{2}\right)\right]
$$

where $\kappa^{2} \equiv 16 \pi G_{D}\left(G_{D}\right.$ is the $D$-dimensional Newton's constant), $\alpha, \beta$ and $\gamma$ are constants. The last term proportional to $\gamma$ is nothing but the Gauss-Bonnet term, which is a surface term in four space-time dimensions.

Now let us expand the metric around a flat Minkowski background $\eta_{\mu \nu}$ as $g_{\mu \nu}=\eta_{\mu \nu}+h_{\mu \nu}$ and keep only quadratic fluctuations in the action. It is convenient to express each term in the action in terms of the spin projection operators:

$$
\begin{aligned}
\mathcal{L}_{E H} & \equiv \sqrt{-g} R \\
& =\frac{1}{4} h^{\mu \nu}\left[P^{(2)}-(D-2) P^{(0, s)}\right]_{\mu \nu, \rho \sigma} \square h^{\rho \sigma},
\end{aligned}
$$

\footnotetext{
${ }^{3}$ The space-time indices $\mu, \nu, \cdots$ run over $0,1, \cdots, D-1$. We take the metric signature $(-,+, \cdots,+)$ and follow the notation and conventions of the textbook of MTW [21].
} 


$$
\begin{aligned}
\mathcal{L}_{\alpha} & \equiv \alpha \sqrt{-g} R^{2} \\
& =\alpha(D-1) h^{\mu \nu} P_{\mu \nu, \rho \sigma}^{(0, s)} \square^{2} h^{\rho \sigma}, \\
\mathcal{L}_{\beta} & \equiv \beta \sqrt{-g} R_{\mu \nu} R^{\mu \nu} \\
& =\beta \frac{1}{4} h^{\mu \nu}\left[P^{(2)}+D P^{(0, s)}\right]_{\mu \nu, \rho \sigma} \square^{2} h^{\rho \sigma}, \\
\mathcal{L}_{\gamma} & \equiv \gamma \sqrt{-g}\left(R_{\mu \nu \rho \sigma} R^{\mu \nu \rho \sigma}-4 R_{\mu \nu} R^{\mu \nu}+R^{2}\right) \\
& =0
\end{aligned}
$$

where in evaluating the last $\mathcal{L}_{\gamma}$ we have used the relation

$$
\sqrt{-g} R_{\mu \nu \rho \sigma} R^{\mu \nu \rho \sigma}=h^{\mu \nu}\left[P^{(2)}+P^{(0, s)}\right]_{\mu \nu, \rho \sigma} \square^{2} h^{\rho \sigma} .
$$

The spin projection operators in $D$ space-time dimensions, $P^{(2)}, P^{(1)}, P^{(0, s)}, P^{(0, w)}, P^{(0, s w)}$ and $P^{(0, w s)}$ form a complete set in the space of second rank symmetric tensors and are defined as $[12]$

$$
\begin{aligned}
P_{\mu \nu, \rho \sigma}^{(2)} & =\frac{1}{2}\left(\theta_{\mu \rho} \theta_{\nu \sigma}+\theta_{\mu \sigma} \theta_{\nu \rho}\right)-\frac{1}{D-1} \theta_{\mu \nu} \theta_{\rho \sigma} \\
P_{\mu \nu, \rho \sigma}^{(1)} & =\frac{1}{2}\left(\theta_{\mu \rho} \omega_{\nu \sigma}+\theta_{\mu \sigma} \omega_{\nu \rho}+\theta_{\nu \rho} \omega_{\mu \sigma}+\theta_{\nu \sigma} \omega_{\mu \rho}\right) \\
P_{\mu \nu, \rho \sigma}^{(0, s)} & =\frac{1}{D-1} \theta_{\mu \nu} \theta_{\rho \sigma} \\
P_{\mu \nu, \rho \sigma}^{(0, w)} & =\omega_{\mu \nu} \omega_{\rho \sigma} \\
P_{\mu \nu, \rho \sigma}^{(0, s w)} & =\frac{1}{\sqrt{D-1}} \theta_{\mu \nu} \omega_{\rho \sigma} \\
P_{\mu \nu, \rho \sigma}^{(0, w s)} & =\frac{1}{\sqrt{D-1}} \omega_{\mu \nu} \theta_{\rho \sigma} .
\end{aligned}
$$

Here the transverse operator $\theta_{\mu \nu}$ and the longitudinal operator $\omega_{\mu \nu}$ are defined as

$$
\begin{aligned}
\theta_{\mu \nu} & =\eta_{\mu \nu}-\frac{1}{\square} \partial_{\mu} \partial_{\nu}=\eta_{\mu \nu}-\omega_{\mu \nu}, \\
\omega_{\mu \nu} & =\frac{1}{\square} \partial_{\mu} \partial_{\nu} .
\end{aligned}
$$

It is straightforward to show that the spin projection operators satisfy the orthogonality relations

$$
\begin{aligned}
P_{\mu \nu, \rho \sigma}^{(i, a)} P_{\rho \sigma, \lambda \tau}^{(j, b)} & =\delta^{i j} \delta^{a b} P_{\mu \nu, \lambda \tau}^{(i, a)}, \\
P_{\mu \nu, \rho \sigma}^{(i, a b)} P_{\rho \sigma, \lambda \tau}^{(j, c d)} & =\delta^{i j} \delta^{b c} P_{\mu \nu, \lambda \tau}^{(i, a)}, \\
P_{\mu \nu, \rho \sigma}^{(i, a)} P_{\rho \sigma, \lambda \tau}^{(j, b c)} & =\delta^{i j} \delta^{a b} P_{\mu \nu, \lambda \tau}^{(i, a c)}, \\
P_{\mu \nu, \rho \sigma}^{(i, a b)} P_{\rho \sigma, \lambda \tau}^{(j, c)} & =\delta^{i j} \delta^{b c} P_{\mu \nu, \lambda \tau}^{(i, a c)},
\end{aligned}
$$


with $i, j=0,1,2$ and $a, b, c, d=s, w$ and the tensorial relation

$$
\left[P^{(2)}+P^{(1)}+P^{(0, s)}+P^{(0, w)}\right]_{\mu \nu, \rho \sigma}=\frac{1}{2}\left(\eta_{\mu \rho} \eta_{\nu \sigma}+\eta_{\mu \sigma} \eta_{\nu \rho}\right)
$$

Using the relations (2), the action (1) takes the form

$$
S=\int d^{D} x \frac{1}{4} h^{\mu \nu} \mathcal{O}_{\mu \nu, \rho \sigma} h^{\rho \sigma}
$$

where $\mathcal{O}_{\mu \nu, \rho \sigma}$ is defined as

$$
\mathcal{O}_{\mu \nu, \rho \sigma}=\square\left[\left(\frac{1}{\kappa^{2}}+\beta \square\right) P^{(2)}+\left\{-\frac{1}{\kappa^{2}}(D-2)+(4 \alpha(D-1)+\beta D) \square\right\} P^{(0, s)}\right]_{\mu \nu, \rho \sigma} .
$$

Then, the graviton propagator is essentially obtained by inverting each spin block

$$
\begin{gathered}
\mathcal{O}_{\mu \nu, \rho \sigma}^{-1}=\frac{1}{\square}\left[\frac{1}{\frac{1}{\kappa^{2}}+\beta \square} P^{(2)}+\frac{1}{-\frac{1}{\kappa^{2}}(D-2)+(4 \alpha(D-1)+\beta D) \square} P^{(0, s)}\right]_{\mu \nu, \rho \sigma} \\
=\kappa^{2}\left[\frac{P^{(2)}-\frac{1}{D-2} P^{(0, s)}}{\square}-\frac{1}{\square+\frac{1}{\beta \kappa^{2}}} P^{(2)}+\frac{1}{D-2} \frac{1}{\square-\frac{D-2}{\kappa^{2}(4 \alpha(D-1)+\beta D)}} P^{(0, s)}\right]_{\mu \nu, \rho \sigma} .
\end{gathered}
$$

Recall that the combination $P^{(2)}-\frac{1}{D-2} P^{(0, s)}$ is parallel to that in four dimensions [22] and the negative sign for the massless pole with $P^{(0, s)}$ does not impose any problem [12].

This structure of the graviton propagator clearly indicates why it is difficult to construct a unitary gravitational theory for the massive graviton in a general space-time dimension. One point which we immediately notice is that the negative sign for the massive pole $\square=-\frac{1}{\beta \kappa^{2}}$ corresponds to a ghost with negative norm, so in order to avoid this massive ghost, we have to impose the condition $\beta=0$, implying that $R_{\mu \nu}^{2}$ term is not allowed to be involved in the action up to the Gauss-Bonnet term. On the other hand, the remaining two poles, massless and massive poles, correspond to spin 2 and 0 unitary modes. (Of course, we have to impose the further conditon $\alpha>0$ in order to avoid tachyons.) In fact, with the vanishing $R_{\mu \nu}^{2}$ term, up to the Gauss-Bonnet term, the action (1) is reduced to $R+R^{2}$, which is known to exactly coincide with Einstein's general relativity with a minimally coupled massive scalar field [23]. In this way, it turns out to be difficult to construct a unitary massive gravity model with helicity \pm 2 massive graviton modes within the framework of the higher-derivative gravity at least in an arbitrary space-time dimension.

However, by inspection, we notice that if the the gravitational coupling constant $\kappa^{2}$ were negative, the residue at the pole $\square=-\frac{1}{\beta \kappa^{2}}$ would become positive whereas those at the massless $\square=0$ and massive poles $\square=\frac{D-2}{\kappa^{2}(4 \alpha(D-1)+\beta D)}$ do negative, thereby implying that the modes corresponding to the former pole and the latter two poles are respectively unitary modes with positive norm and ghosts with negative norm. At this stage, a nice thing happens in three dimensions. Namely, in three dimensions, it turns out that massless graviton modes are non-dynamical [12] so we can neglect the massless graviton whatever its norm is positive or negative. The remaining problem is therefore cast to a problem how to deal with the massive 
scalar mode with negative norm. This problem is overcome by selecting the constants $\alpha, \beta$ in such a way that they satisfy the relation

$$
\left.(4 \alpha(D-1)+\beta D)\right|_{D=3}=8 \alpha+3 \beta=0 .
$$

After all, we arrive at a unitary massive gravity action in three dimensions which has been recently found by Bergshoeff et al. [11]

$$
S=\int d^{3} x \frac{1}{\kappa^{2}} \sqrt{-g}\left[-R+\frac{1}{M^{2}}\left(R_{\mu \nu} R^{\mu \nu}-\frac{3}{8} R^{2}\right)\right]
$$

where we have set $\beta \equiv \frac{1}{\kappa^{2} M^{2}}$ because of $\beta>0$. Note that we have replaced $\kappa^{2}$ in (1) with $-\kappa^{2}$ in (12) in order to make the sign for the Einstein-Hilbert term negative.

\section{Massive gravity with the Pauli-Fierz mass term}

Now we shall add the Pauli-Fierz mass term to the new massive gravity in three dimensions and examine the effect on the graviton propagator.

For this purpose, let us begin with the action

$$
S=\int d^{3} x\left[\frac{1}{\kappa^{2}} \sqrt{-g}\left\{-R+\frac{1}{M^{2}}\left(R_{\mu \nu} R^{\mu \nu}-\frac{3}{8} R^{2}\right)\right\}-\frac{m^{2}}{4}\left(h_{\mu \nu} h^{\mu \nu}-h^{2}\right)\right],
$$

where $h \equiv \eta^{\mu \nu} h_{\mu \nu}$ and we shall set $\kappa^{2}=1$ henceforth. First, let us note that the Pauli-Fierz mass term can be rewritten by the spin projection operators as

$$
\begin{aligned}
\mathcal{L}_{P F} & \equiv-\frac{m^{2}}{4}\left(h_{\mu \nu} h^{\mu \nu}-h^{2}\right) \\
& =\frac{m^{2}}{2} h^{\mu \nu}\left[-\frac{1}{2} P^{(2)}-\frac{1}{2} P^{(1)}+\frac{1}{2} P^{(0, s)}+\frac{1}{\sqrt{2}}\left(P^{(0, s w)}+P^{(0, w s)}\right)\right]_{\mu \nu, \rho \sigma} h^{\rho \sigma} .
\end{aligned}
$$

Thus, the quadratic part of the action (13) is expressed in term of the spin projection operators like

$$
S=\int d^{3} x \frac{1}{2} h^{\mu \nu} \mathcal{P}_{\mu \nu, \rho \sigma} h^{\rho \sigma}
$$

where $\mathcal{P}_{\mu \nu, \rho \sigma}$ is defined as

$$
\begin{aligned}
\mathcal{P}_{\mu \nu, \rho \sigma} & =\left[\frac{1}{2}\left(\frac{1}{M^{2}} \square^{2}-\square-m^{2}\right) P^{(2)}-\frac{m^{2}}{2} P^{(1)}+\frac{1}{2}\left(\square+m^{2}\right) P^{(0, s)}\right. \\
& \left.+\frac{m^{2}}{\sqrt{2}}\left(P^{(0, s w)}+P^{(0, w s)}\right)\right]_{\mu \nu, \rho \sigma} .
\end{aligned}
$$

Then, the propagator for $h_{\mu \nu}$ is defined by

$$
<0\left|T\left(h_{\mu \nu}(x) h_{\rho \sigma}(y)\right)\right| 0>=i \mathcal{P}_{\mu \nu, \rho \sigma}^{-1} \delta^{(D)}(x-y),
$$


where using the relation (7), the inverse of the operator $\mathcal{P}$ is easily calculated as

$$
\begin{aligned}
\mathcal{P}_{\mu \nu, \rho \sigma}^{-1} & =\left[\frac{2}{\frac{1}{M^{2}} \square^{2}-\square-m^{2}} P^{(2)}-\frac{2}{m^{2}} P^{(1)}-\frac{\square+m^{2}}{m^{4}} P^{(0, w)}\right. \\
& \left.+\frac{\sqrt{2}}{m^{2}}\left(P^{(0, s w)}+P^{(0, w s)}\right)\right]_{\mu \nu, \rho \sigma} .
\end{aligned}
$$

The expression of the propagator (18) reveals that there are massive poles in the sector of spin 2 graviton modes, which is of form

$$
\begin{aligned}
I & \equiv \frac{1}{M^{2}} \square^{2}-\square-m^{2} \\
& =\frac{1}{M^{2}}\left(\square-\omega_{+}\right)\left(\square-\omega_{-}\right),
\end{aligned}
$$

where $\omega_{ \pm} \equiv \frac{1 \pm \sqrt{1+4\left(\frac{m}{M}\right)^{2}}}{2} M^{2}$, which are real numbers such that $\omega_{+}>0, \omega_{-}<0$. In order to understand the physical property of the poles, it is useful to rewrite $\frac{1}{I}$ as

$$
\frac{1}{I}=\frac{1}{\sqrt{1+4\left(\frac{m}{M}\right)^{2}}}\left(\frac{1}{\square-\omega_{+}}-\frac{1}{\square-\omega_{-}}\right),
$$

which shows that there are two massive spin 2 modes. One is the unitary mode of positive mass $\omega_{+}$with positive norm while the other is the ghost of tachyonic mass $\omega_{-}$with negative norm. Thus in the model at hand the existence of this tachyonic ghost induces the violation of both unitarity and causality, so that adding the Pauli-Fierz mass term to the new massive gravity theory in three dimensions is not permitted from the physical requirements of unitarity and causality.

\section{Incorporation of 'gravitational' Chern-Simons term}

Next, in this section, we wish to consider the most general situation where three distinct massgenerating terms, those are, the Pauli-Fierz, higher-derivative, and 'gravitational' ChernSimons terms, coexist with the Einstein-Hilbert action in three dimensions. In particular, we are interested in the question whether or not the presence of the gravitational Chern-Simons term could rectify the impossibility of adding the Pauli-Fierz mass term to the new massive gravity model. Actually, it has been already pointed out that in the case of the topological massive gravity with the Pauli-Fierz mass term, there is a parameter region where ghosts and tachyons are simultaneously excluded and we have three massive (not tachyonic) excitations with positive norm $[18,19]$.

The most general action which includes three mass-generating terms is given by

$$
\begin{aligned}
S & =\int d^{3} x\left[\sqrt{-g}\left\{-R+\frac{1}{M^{2}}\left(R_{\mu \nu} R^{\mu \nu}-\frac{3}{8} R^{2}\right)\right\}-\frac{m^{2}}{4}\left(h_{\mu \nu} h^{\mu \nu}-h^{2}\right)\right. \\
& \left.+\frac{1}{\mu} \varepsilon^{\mu \nu \rho} \Gamma_{\mu \lambda}^{\sigma}\left(\partial_{\nu} \Gamma_{\sigma \rho}^{\lambda}+\frac{2}{3} \Gamma_{\nu \tau}^{\lambda} \Gamma_{\rho \sigma}^{\tau}\right)\right],
\end{aligned}
$$


where the coefficient $\mu$ in front of the gravitational Chern-Simons term is a constant. In order to accommodate with this topological term, one has to introduce additional two operators to the whole spin projection operators [18]

$$
\begin{aligned}
& S_{1 \mu \nu, \rho \sigma}=\frac{1}{4} \square\left(\varepsilon_{\mu \rho \lambda} \partial_{\sigma} \omega_{\nu}^{\lambda}+\varepsilon_{\mu \sigma \lambda} \partial_{\rho} \omega_{\nu}^{\lambda}+\varepsilon_{\nu \rho \lambda} \partial_{\sigma} \omega_{\mu}^{\lambda}+\varepsilon_{\nu \sigma \lambda} \partial_{\rho} \omega_{\mu}^{\lambda}\right), \\
& S_{2 \mu \nu, \rho \sigma}=-\frac{1}{4} \square\left(\varepsilon_{\mu \rho \lambda} \eta_{\sigma \nu}+\varepsilon_{\mu \sigma \lambda} \eta_{\rho \nu}+\varepsilon_{\nu \rho \lambda} \eta_{\sigma \mu}+\varepsilon_{\nu \sigma \lambda} \eta_{\rho \mu}\right) \partial^{\lambda} .
\end{aligned}
$$

These operators together with the spin projection operators satisfy the following relations

$$
\begin{aligned}
S_{1} S_{1} & =\frac{1}{4} \square^{3} P^{(1)}, \\
S_{1} S_{2} & =S_{2} S_{1}=-\frac{1}{4} \square^{3} P^{(1)}, \\
S_{2} S_{2} & =\square^{3}\left(P^{(2)}+\frac{1}{4} P^{(1)}\right), \\
P^{(1)} S_{1} & =S_{1} P^{(1)}=S_{1}, \\
P^{(1)} S_{2} & =S_{2} P^{(1)}=-S_{1}, \\
P^{(2)} S_{2} & =S_{2} P^{(2)}=S_{1}+S_{2},
\end{aligned}
$$

where the matrix indices are to be understood.

As before, we write out the quadratic fluctuations in $h_{\mu \nu}$ in the action (21) whose result reads

$$
S=\int d^{3} x \frac{1}{2} h^{\mu \nu} \mathcal{Q}_{\mu \nu, \rho \sigma} h^{\rho \sigma}
$$

where $\mathcal{Q}_{\mu \nu, \rho \sigma}$ is defined as

$$
\begin{aligned}
\mathcal{Q}_{\mu \nu, \rho \sigma} & =\left[\frac{1}{2}\left(\frac{1}{M^{2}} \square^{2}-\square-m^{2}\right) P^{(2)}-\frac{m^{2}}{2} P^{(1)}+\frac{1}{2}\left(\square+m^{2}\right) P^{(0, s)}\right. \\
& \left.+\frac{m^{2}}{\sqrt{2}}\left(P^{(0, s w)}+P^{(0, w s)}\right)+\frac{1}{\mu}\left(S_{1}+S_{2}\right)\right]_{\mu \nu, \rho \sigma} .
\end{aligned}
$$

The inverse matrix of $\mathcal{Q}_{\mu \nu, \rho \sigma}$, which is proportional to the graviton propagator, is calculated as

$$
\begin{aligned}
\mathcal{Q}_{\mu \nu, \rho \sigma}^{-1} & =\left[\frac{2\left(\frac{1}{M^{2}} \square^{2}-\square-m^{2}\right)}{\left(\frac{1}{M^{2}} \square^{2}-\square-m^{2}\right)^{2}-\frac{4}{\mu^{2}} \square^{3}} P^{(2)}-\frac{2}{m^{2}} P^{(1)}-\frac{\square+m^{2}}{m^{4}} P^{(0, w)}\right. \\
& \left.+\frac{\sqrt{2}}{m^{2}}\left(P^{(0, s w)}+P^{(0, w s)}\right)-\frac{4}{\mu} \frac{1}{\left(\frac{1}{M^{2}} \square^{2}-\square-m^{2}\right)^{2}-\frac{4}{\mu^{2}} \square^{3}}\left(S_{1}+S_{2}\right)\right]_{\mu \nu, \rho \sigma} .
\end{aligned}
$$

It is easy to see that this expression reduces to (18) in the limit of $\mu \rightarrow \infty$ as required.

As opposed to the previous case without the gravitational Chern-Simons term (18), there appears quartic pole in the sectors of $P^{(2)}, S_{1}$ and $S_{2}$. Thus, there might be a certain parameter 
region where a unitary and tachyon-free massive gravity theory exists. In order to examine this possibility, let us consider the pole structure

$$
\begin{aligned}
J & \equiv\left(\frac{1}{M^{2}} \square^{2}-\square-m^{2}\right)^{2}-\frac{4}{\mu^{2}} \square^{3} \\
& =\left(\frac{1}{M^{2}} \square^{2}-\square-m^{2}+\frac{2}{\mu} \square^{\frac{3}{2}}\right)\left(\frac{1}{M^{2}} \square^{2}-\square-m^{2}-\frac{2}{\mu} \square^{\frac{3}{2}}\right) .
\end{aligned}
$$

The necessary condition that there is no ghost is given by the condition such that the quartic equation $J=0$ has no real solution. It then turns out that the equation $J=0$ has indeed real solutions for any real value of $M, m$ and $\mu$. Hence, even in this case where the gravitational Chern-Simons term is added to the Pauli-Fierz term plus the new massive gravity theory, there is no physically plausible, unitary massive gravity theory. In this sense, the Pauli-Fierz mass term is not allowed to exist in the new massive gravity model in three dimensions. In this context, let us recall that the Pauli-Fierz massive gravity makes sense only as a free theory holding in the quadratic approximation level in $h_{\mu \nu}$ so the theory is not diffeomorphisminvariant but Lorentz-invariant. On the other hand, the new massive gravity is an interacting and diffeomorphism-invariant theory, so the theory might not admit the existence of the diffeomorphism-noninvariant Pauli-Fierz term.

\section{References}

[1] M. Fierz and W. Pauli, "On Relativistic Wave Equations for Particles of Arbitrary Spin in an Electromagnetic Field", Proc. Roy. Soc. Lond. A173 (1939) 211.

[2] R. Percacci, "The Higgs Phenomenon in Quantum Gravity", Nucl. Phys.B353 (1991) 271, arXiv:0712.3545 [hep-th].

[3] Z. Kakushadze and P. Langfelder, "Gravitational Higgs Mechanism", Mod. Phys. Lett.A15 (2000) 2265, arXiv:hep-th/0011245.

[4] M. Porrati, "Higgs Phenomenon for 4-D Gravity in Anti de Sitter Space", JHEP0204 (2002) 058, arXiv:hep-th/0112166.

[5] I. Kirsch, "A Higgs Mechanism for Gravity", Phys. Rev.D72 (2005) 024001, arXiv:hepth/0503024.

[6] G. 't Hooft, "Unitarity in the Brout-Englert-Higgs Mechanism for Gravity", arXiv:0708.3184 [hep-th].

[7] Z. Kakushadze, "Gravitational Higgs Mechanism and Massive Gravity", Int. J. Mod. Phys.A23 (2008), arXiv:0709.1673 [hep-th]. 
[8] I. Oda, "Gravitational Higgs Mechanism with a Topological Term", Advanced Studies in Theoretical Physics 2 (2008) 261, arXiv:0709.2419 [hep-th].

[9] M. Maeno and I. Oda, "Classical Solutions of Ghost Condensation Models", Mod. Phys. Lett.B22 (2009) 3025, arXiv:0801.0827 [hep-th].

[10] M. Maeno and I. Oda, "Massive Gravity in Curved Cosmological Backgrounds", Int. J. Mod. Phys.A24 (2009) 81, arXiv:0808.1394 [hep-th].

[11] E.A. Bergshoeff, O. Hohm and P.A. Townsend, "Massive Gravity in Three Dimensions", arXiv:0901.1766 [hep-th].

[12] M. Nakasone and I. Oda, "On Unitarity of Massive Gravity in Three Dimensions", arXiv:0902.3531 [hep-th].

[13] G. Clement, "Warped AdS3 Black Holes in New Massive Gravity", arXiv:0902.4634 [hepth].

[14] Y. Liu and Y. Sun, "Note on New Massive Gravity in AdS3", arXiv:0903.0536 [hep-th].

[15] S. Deser, R. Jackiw and S. Templeton, "Topologically Massive Gauge Theories", Ann. of Phys.140 (1982) 372 [Erratum-ibid. 137B (1988 APNYA, 281, 409-449. 2000) 406.1988 APNYA, 281, 409].

[16] I. Oda, "Higgs Mechanism with a Topological Term", Mod. Phys. Lett.A16 (2001) 1493, arXiv:hep-th/0104085.

[17] I. Oda, "Higgs Mechanism in the Presence of a Topological Term", Int. J. Mod. Phys.A17 (2002) 89, arXiv:hep-th/0106210.

[18] C. Pinheiro, G.O. Pires and N. Tomimura, "Some Quantum Aspects of Threedimensional Einstein-Chern-Simons-Proca Massive Gravity", Il Nuovo Cim.111B (1996) 1023.

[19] S. Deser and B. Tekin, "Massive, Topologically Massive, Models", Class. Quantum. Grav.19 (2002) L97-L100, arXiv:hep-th/0203273.

[20] K.S. Stelle, "Renormalization of Higher-derivative Quantum Gravity", Phys. Rev.D16 (1977) 953.

[21] C.W. Misner, K.S. Thorne and J.A. Wheeler, "Gravitation", W H Freeman and Co (Sd), 1973.

[22] P. van Nieuwenhuizen, "Supergravity", Phy. Rep. 68 (1981) 189; and references therein.

[23] H.J. Schmidt, "Fourth Order Gravity: Equations, History, and Applications to Cosmology", Int. J. Geom. Meth. Mod. Phys. 4 (2007) 209, arXiv:gr-qc/0602017. 